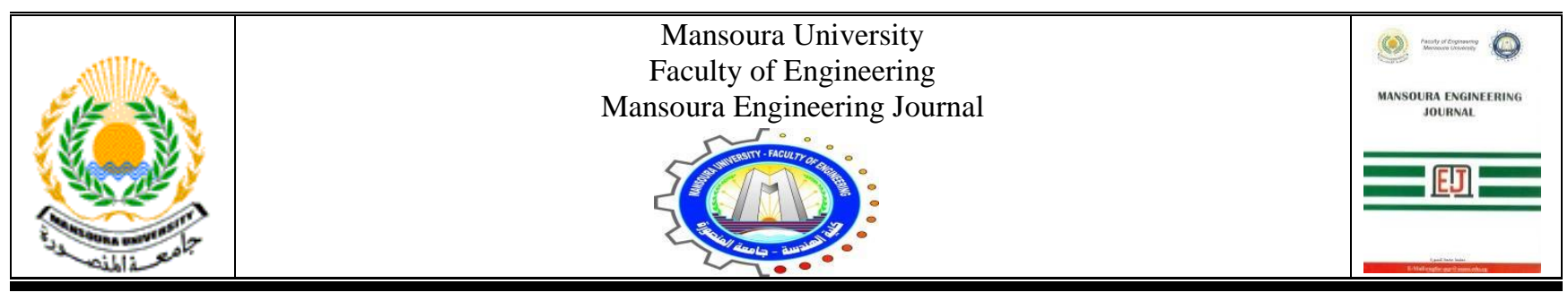

\title{
MRI Brain Tumor Segmentation Using Deep Learning
}

\author{
Shaimaa E. Nassar, Mohamed A. El-Azim and Ahmed Elnakib
}

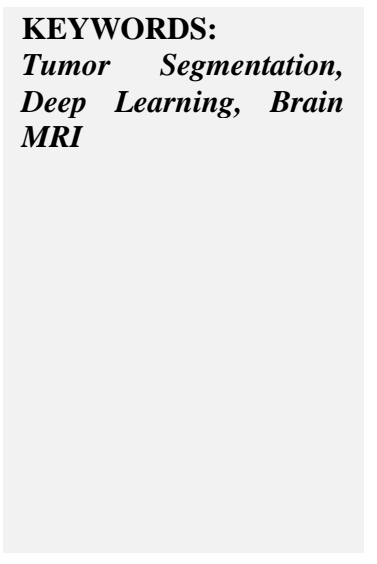

\begin{abstract}
This work presents a method for classification and segmentation of brain tumors based on deep learning analysis of brain contrast T1 (T1c) MR images. To achieve this goal, three different deep learning networks are investigated i.e., U-Net, VGG16-Segnet, and DeepLabv3+ models. In addition, the integration of the 3D narrow-band information of the MRI volumes is imported to the input of the Convolutional Neural Network (CNN) to describe more accurately the tumor anatomy. Experimentations are performed on the MICCAI'2018 High Grade Glioma (HGG) subset of the Brain Tumor Segmentation (BraTS) Challenge, composed of 210 brain T1c MRI volumes, each of 155 cross-sections. Among the three investigated CNNs, DeepLabv3+ network achieves the highest Dice Similarity Coefficients (DSC) of 91.2\%, 92.5\%, 94.6\% for the segmentation of the Enhancing Tumor (ET), the Tumor Core (TC), and the Whole Tumor (WT), respectively. Comparison with the related work confirms the advantages of the proposed system.
\end{abstract}

\section{INTRODUCTION}

$\mathrm{B}$ RAIN tumor is an epidemic causes of cancer death. In USA, 700.000 people are diagnosed with brain tumors (80\% benign and 20\% malignant) [1]. In 2020, the American Cancer Society (ACS) for brain tumor estimated about 23,890 malignant tumors of the brain and around 18,020 deaths from malignant brain tumors [2]. Accurate segmentation and quantitative analysis of brain tumor is critical for tumor diagnosis and treatment planning.

Magnetic resonance imaging (MRI) is usually used for brain tumor segmentation and classification. Since manual segmentation is a difficult and a time consuming task, the

Received: (5 September, 2020) - Revised: (9 December, 2020) - Accepted: (20 December, 2020)

Shaimaa E. Nassar, is with the Electronics and Communications Engineering Department, Faculty of Engineering, Mansoura University, 35516 Mansoura City, Egypt (e-mail: shaimaaelsabahy@yahoo.com)

Mohamed A. El-Azim, is with the Electronics and Communications Engineering Department, Faculty of Engineering, Mansoura University, 35516 Mansoura City, Egypt (e-mail: mazim12@yahoo.com)

* Corresponding author: A. Elnakib, is with the Electronics and Communications Engineering Department, Faculty of Engineering, Mansoura University, 35516 Mansoura City, Egypt (e-mail: nakib@mans.edu.eg) automated segmentation using Computer Aided Diagnostic (CAD) systems, to assist the radiologists for brain tumor segmentation, is a widely investigated open research problem.

Throughout literature, different methodologies have been investigated for brain tumor segmentation. These methods can be categorized as traditional methods (discriminative or generative) and deep learning methods. Below, the related work of each of these categories will be described

Discriminative methods attempt to extract discriminative features followed by a classifier in order to perform classification [3]. For example, Dandil et al. [4] used a spatialFuzzy C-Means (FCM) classifier based on the image intensity feature to achieve an accuracy of 0.91 , a sensitivity of 0.91 sensitivity, and a specificity of 0.95 for "WT" segmentation. Tustison et al. [5] investigated different features, including the intensity, the geometry, and the asymmetry features, extracted form multiple MRI modalities. A random forest classifier, based on the asymmetry-related features, achieved the best performance on BraTS 2013 database [6], i.e., DSCs of 0.87, 0.78 , and 0.74 for "WT", "TC", and "ET" components, respectively. However, the discriminative methods rely on the quality of the extracted features, which may not represent well the tumor segmentation problem.

Generative methods use atlases of healthy tissues to 
extract the unknown tumor compartments on test images, by the aiding of prior knowledge, e.g., location and the spatial extent of healthy tissues. For example, Kuwon et al. [7] used an atlas generation method for the segmentation of multifocal tumors, using the BraTS 2013 database. They have achieved accuracies of $0.86,0.79$, and 0.59 for "WT", "TC", and "ET" components, respectively. However, these methods required a high quality registration of the test images to the atlas, which is a complicated and a computationally expensive task.

Deep learning methods use Convolutional Neural Networks (CNN), arranged in convolutional layers to extract deep learning features, followed by classification layers in order to output the segmentation labels. These methods have shown ability to segment and classify the brain tumor with high accuracies. For example, Shaocheng et al. [8] used a 10layer U-Net architecture, trained on 2-D slices, extracted from patient volumes, using the BraTS 2018 dataset [9]. They achieved DSC coefficients of $0.91,0.83$, and 0.80 for "WT", "TC", and "ET" components, respectively. Mohammad et al. [10] used two pathway CNN architecture with two streams: local pathway and the global pathway. They have achieved $0.88,0.79$, and 0.73 for "WT", "TC", and "ET" components, respectively. The main limitation of the previous work are:

- Current discriminative methods suffer from insufficient features that can represent the brain cancer problem, leading to lower accuracies

- Current generative methods suffer from the computationally expensive registration tasks. In addition, the built atlas may not represent well the image population.

- Current Deep learning methods suffer from the computationally expensive cost for training the CNN layers. In addition, the selection of the best number of layers and the best number of neurons per each layer is still an open research problem.

To overcome these limitations, A CAD system for brain tumor segmentation based on deep transfer learning is proposed, as shown in Fig. (1). The proposed system shows an ability to accurately segment the brain tumors, evidenced by the obtained high DSC metrics. The main contributions of this work are as follows:

- Applying transfer learning of well-known architectures, where the convolutional layers are transferred from pertained models and only the classification layers are trained with the new data (BraTs), minimizing the training overhead

- Investigating different recent $\mathrm{CNN}$ architectures for brain tumor segmentation, i.e., U-Net, VGG16-Segnet, and DeepLabv3+.

- Utilization of a "3D narrow-band" information of MRI data to more accurately describe the tumor anatomy.

- Performance evaluation using the challenging MICCAI'2018 BraTS database

This manuscript is organized as follows. Section II illustrates in detail the proposed system for brain tumor segmentation and classification. Section III summarizes the proposed system results as well as the comparative results to the current state-of-the-art techniques. Finally, section IV concludes the manuscript.

\section{Materials AND Methods}

The proposed framework, shown in Fig. (1), consists of three processing stages. First, 3D narrow-band volume is selected, around the target image. Second, the features are extracted using the CNN model. Finally, a pixel-wise classifier is used to classify each pixel to one of four labels: edema (ED), tumor core (TC), enhancing tumor (ET), and background (BG).

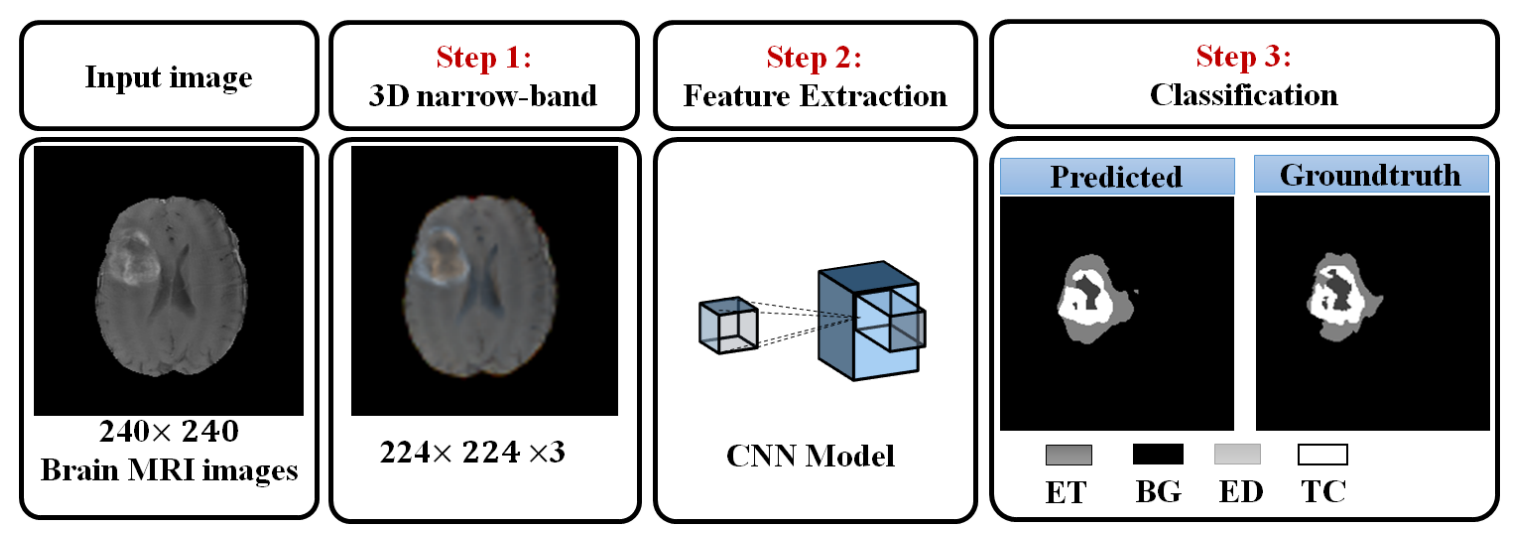

Fig.1: Proposed framework for Brain tumor segmentation composed of three stages: 3D narrow-band selection, feature extraction, and classification.

\section{A. Collected Database (BraTS'2018)}

BraTS'2018 [9] is used throughout this study. This data is composed of 210 high-grade glioma (HGG) and 75 low-grade glioma (LGG) patient datasets. Each dataset contains T1, T1 post contrast (T1c), T2, and Fluid Attenuated Inverse
Recovery (FLAIR) MR volumes, alone with an expert tumor segmentation. Each scan is a continuous 3D volume of $1552 \mathrm{D}$ slices of size 240x240. Each brain tumor is manually delineated into 4 classes: background, edema (ED), necrotic/non-enhancing core (NCR/NET), and enhancing tumor core (ET). For tumor segmentation, we consider only 
the post T1c contrast HGG MRI data to identify the WT (ED $+\mathrm{NCR} / \mathrm{NET}+\mathrm{ET}), \mathrm{TC}(\mathrm{NCR} / \mathrm{NET}+\mathrm{ET})$ and the ET of the high-grade brain tumor. The tumor is more clear in the post T1c contrast data, therefore, T1c MR images are popular used for brain tumor segmentation [11],[12]. More details can be found in [9].

\section{B. 3D Narrow-Band Selection}

Since the proposed system aims to segment and classify tumors, only images that contain tumors are selected, to avoid redundant training operations. In most cases, the tumor appears in around $66 \%$ of the volume cross-sections. Each image is normalized between $0-1$ and resized to the standard size of the CNN model. A "3D narrow-band" filter is applied to each input image, by the insertion of the target image at the centralized input channel, with its previous and next slices inserted to each side channel, as shown in Fig. (2)'

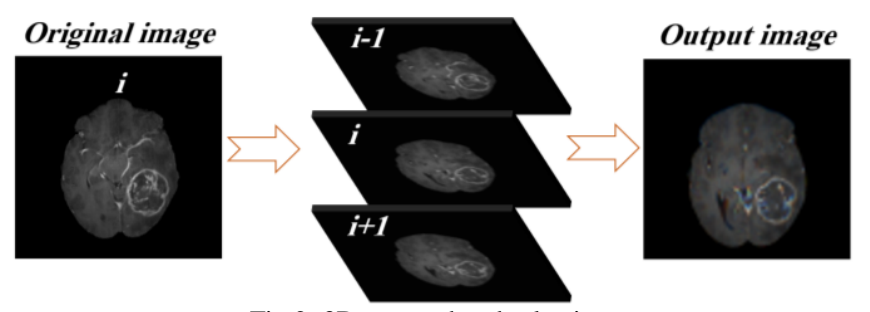

Fig.2: 3D narrow band selection.

\section{Feature extraction}

Three pre-trained CNN models are investigated to extract tumor features, i.e., U-Net [13], Visual Geometry Group Segmentation Network (VGG16-Segnet [14]) and DeepLabV3+ [15]. U-Net consists of a contracting path and an expanding path and yields a u-shaped architecture, as shown in Fig. (3).

The contracting path made feature extraction and downsampling, and expanding path made decoding. VGG16SegNet consists of an encoder network, a corresponding decoder network followed by a pixel-wise classification layer. The architecture of the encoder network is topologically identical to the 13 convolutional layers in the VGG16 network [14], as shown in Fig. (4). The role of the decoder network is to map the low resolution (up sampling) encoder feature maps to full input resolution feature maps for pixel-wise classification. All the encoder's weights are initialized by transfer all the pertained weights of the VGG16 model in [16] and ResNet101 in [17], respectively. In the training phase, all the encoder layers and decoder layers are fine trained using the BRaTS data.

On the other hand, the deepLabv3+ [15] model combines two techniques:

- An encoder-decoder pathway
- Atrous Spatial Pyramid Pooling (ASPP) to obtain accurate delineation of object boundaries, as shown in Fig. (5).

The encoder-decoder pathway consists of $(i)$ the pretrained Resnet-101 encoder [17], to extract image features, and (ii) a decoder, to decode the output of the appropriate dimensions. The Atrous convolutions are used to capture the information at different scales. To control the convolution effective field of view, the Atrous used the controlling rate parameter, rate.

The generalized form of Atrous convolutions is given as:

$$
y(n)=\sum_{k} x(n+\text { rate } \times k) w(k)
$$

where $n$ is the location in the output feature map $y, w$ is a convolution filter, and rate is the atrous rate, rate that determines the stride in which the input signal is sampled

The normal convolution is a special case of atrous convolutions with rate $=1$, as shown in Fig. (6).

DeepLab V3+ uses aligned Xception[18], which has the main feature extractor, with the following modifications:

- All max pooling operations are replaced by depthwise separable convolution with striding.

- After each $3 \times 3$ depthwise convolution, extra batch normalization and Rectified Linear Unit (ReLU) activation are added.

- The model depth is increased without changing the entry flow network structure.

The three models are applied (U-Net, VGG16-Segnet, and DeepLabv3+) in the proposed system, since their decoders produce outputs that are of the same dimensions as the input image, which suits the task of segmentation. In addition, they have repeatedly used in similar medical image segmentation tasks, such as skin lesion segmentation [19], Liver lesion segmentation [20], [21], lung segmentation [22],[23], and pathological lymph node segmentation [24].

\section{Classification:}

A pixel-wise classifier is applied after VGG16-Segnet and DeepLabv3+ models to label the segmented output image. The pixel-wise classifier is composed of two layers: a layer of the softmax function and a layer of the pixel-wise classification. The softmax layer is consisted of four softmax nodes per each image pixel, providing the probabilities of the four labels: WT, TC, ET or background, as follows:

$$
\sigma\left(x_{j}\right)=\frac{e^{x_{j}}}{\sum_{j} e^{x_{j}}}
$$

where $x_{j}$ denotes the input at the softmax node $j$ and $\sigma($.$) denotes the output probability of the softmax node. The$ pixel classification layer weights are trained using the BraTS database. Relies on the largest probability of softmax, the pixel-wise classification layer provides the final output label for each pixel to be either WT, TC, ET or background. The algorithmic steps of the proposed system is summarized in algorithm 1. 


\section{Algorithm 1: Proposed Segmentation method}

\begin{tabular}{ll} 
Input: & $\begin{array}{l}\text { Original Image, } \\
\text { Output: }\end{array}$ \\
Labeled Image, BG, ED, ET, and TC \\
\hline Steps: & \\
Step-1 & Preprocess the original image as follows: \\
& Each image is normalized between 0-1 \\
& Apply a 3D narrow-band, as shown in Fig. 2.
\end{tabular}

\begin{tabular}{ll}
\hline Step-2 & Extract features using the investigated CNN models ( U-Net, VGG16-Segnet, and DeepLabv3+) \\
\hline Step-3 & Apply pixel-wise neural network to output the image labels (ED, ET, TC, BG)
\end{tabular}

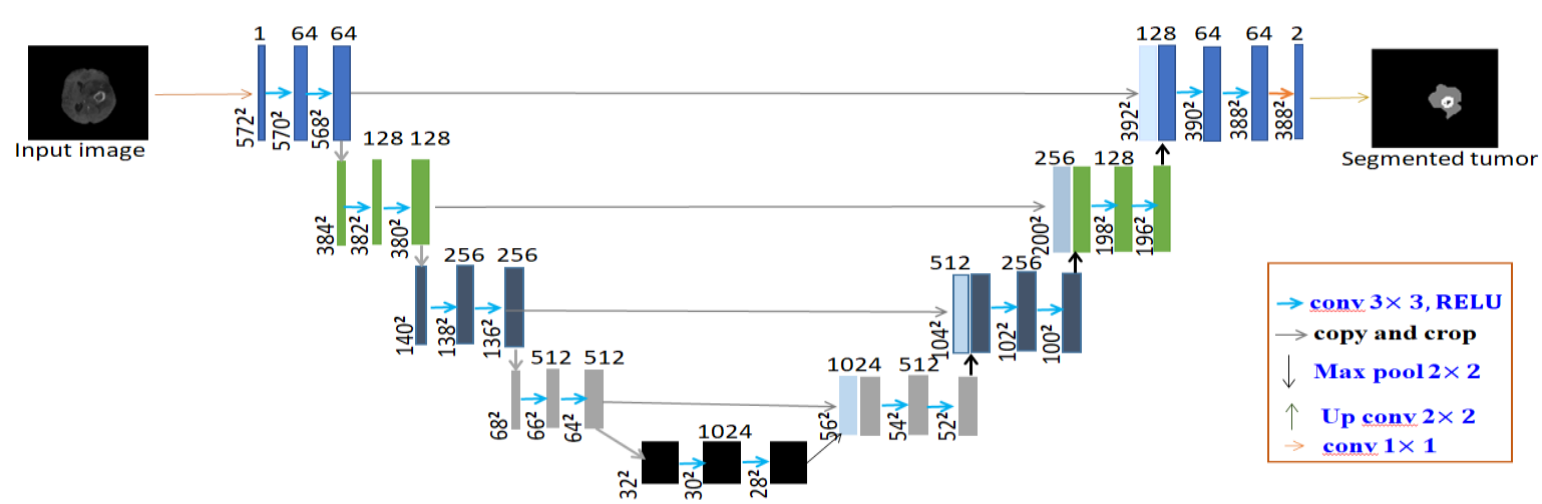

Fig.3: U-Net architecture

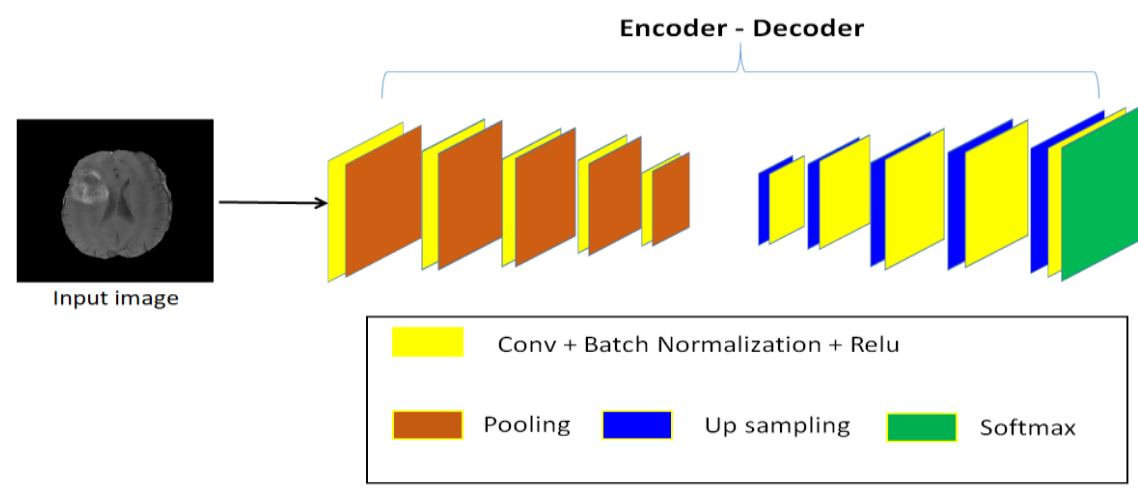

Fig.4: SegNet architecture

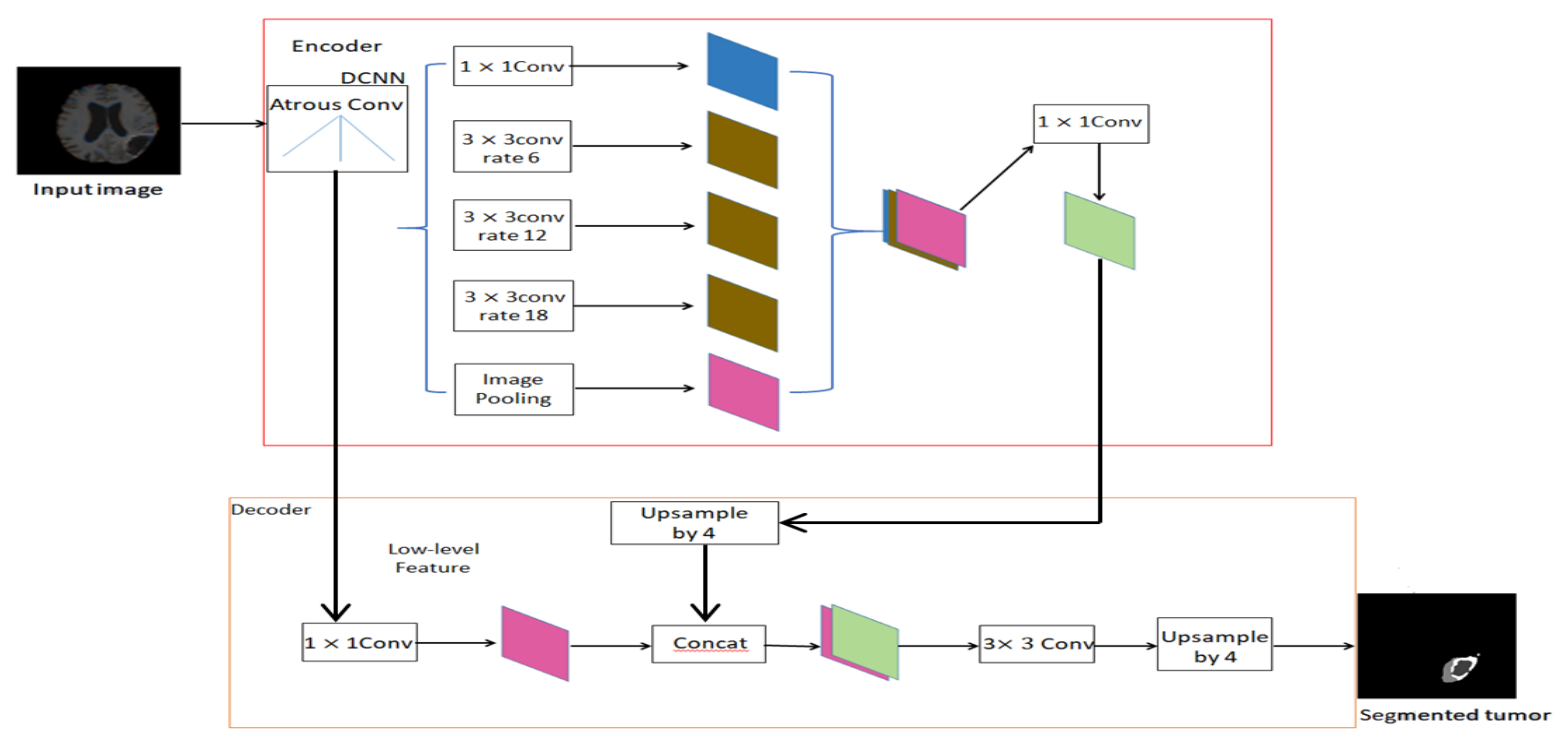

Fig5: Deeplabv3+ architecture 


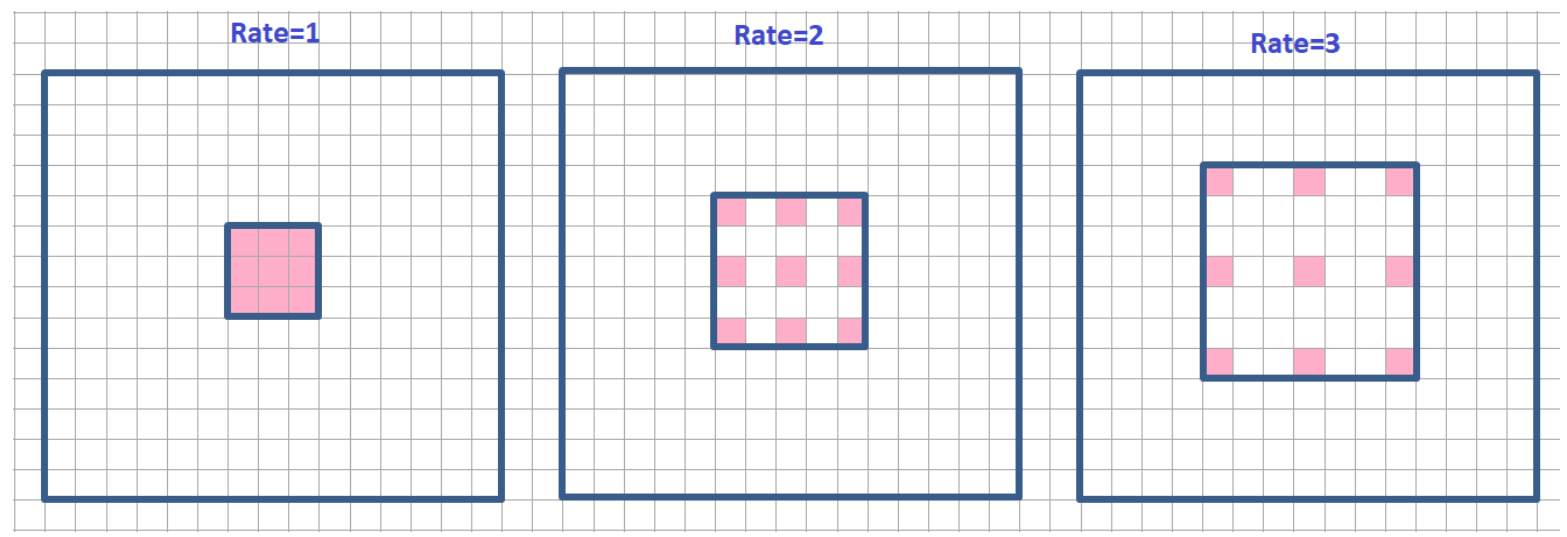

Fig6: Atrous convolutions with different rates, atrous convolutions are able to capture information from a larger effective field of view during the use of the same number of parameters and computational complexity.

\section{RESUlts AND DISCUSSION}

This section illustrates, in details, the experimental setup, results, and related discussions.

\section{A. Performance Metrics}

The accuracy of proposed system evaluated for utilizing $\mathrm{U}$ Net, VGG16-Segnet, and Deeplabv3+ models using the Dice Similarity Coefficient (DSC), the Sensitivity, and the Specificity metrics.

$$
\begin{aligned}
& \mathrm{DSC}=\frac{2 \times \mathrm{TP}}{2 \times \mathrm{TP}+\mathrm{FP}+\mathrm{FN}} \times 100 \% \\
& \text { Sensitivity }=\frac{\mathrm{TP}}{\mathrm{TP}+\mathrm{FN}} \times 100 \% \\
& \text { Specificity }=\frac{\mathrm{TN}}{\mathrm{TP}+\mathrm{FP}} \times 100 \%
\end{aligned}
$$

\section{B. Experimentation Setting}

The CNN models U-Net, VGG16-SegNet, and DeepLabV3+ were trained using the dataset of BRaTS 2018 challenge. The proposed system used 13,181 images of all the 210 scans containing tumor areas of T1c MR modality of HGG (i.e., 10,545 training images (80\% of the images) and 2,636 test images (20\% of images)). ADAM [25] optimizer is used for U-Net training, where the other two models use the stochastic gradient descent with momentum (SGDM) optimizer. Table II summarizes the training options of the utilized CNN models. All training phases are implemented using Matlab@ $2019 b$.

\begin{tabular}{|c|c|c|c|c|c|}
\hline $\begin{array}{l}\text { CNN } \\
\text { model }\end{array}$ & $\begin{array}{l}\text { Input } \\
\text { image }\end{array}$ & och & $\begin{array}{l}\text { Learnin } \\
\text { g Rate }\end{array}$ & $\begin{array}{l}\text { Optimi } \\
\text { zer }\end{array}$ & $\begin{array}{l}\text { \#batch } \\
\text { size }\end{array}$ \\
\hline Unet & $96 \times 96$ & 100 & $10^{-4}$ & ADAM & 16 \\
\hline Vgg16-segnet & $96 \times 96 \times 3$ & 50 & $10^{-4}$ & SGDM & 8 \\
\hline deeplabv3+ & $224 \times 224 \times 3$ & 50 & $10^{-4}$ & SGDM & 8 \\
\hline
\end{tabular}

TABLE II

TRAINING OPTIONS FOR U-NET, VGG16-SEGNET\& DEEPLABV3+

\section{Qualitative Results}

To compare between the efficiency of the three investigated models for brain tumor segmentation, Figure 7 carries visual comparison results for a sample test image. As shown in Fig. 7, deeplabv3+ has achieved the best segmentation results, because of the added value of the embedded Atrous Spatial Pyramid Pooling (ASPP) within the deeplabv3+ model. In addition, adding the 3D narrow band information has further improved the segmentation quality of the deeplabv3+ model. Figure 8 represents confusion matrices of U-Net, VGG16-Segnet, Deeplabv3+ and Deeplabv3+ with narrow-band.

\section{D.Quantitative Results}

To quantify the proposed system, Table III provides detailed brain tumor segmentation results for each utilized deep learning model (U-Net, VGG16-Segnet, and DeepLabv3+). Compatible with the visual results (Fig. 7), the DeepLabv3+ model provides the highest segmentation accuracies. In addition, adding the 3D narrow-band information is able to improve further the accuracies for all segmentation labels. This highlight the advantages of the proposed system using the 3D narrow band information.

\section{E. Comparative Results}

To highlight the advantages and benefits of the proposed system, Table IV compares the achieved results to the related state-of-the-art methods on the BRaTS 2018 challenge database. The proposed system achieves superior performance for tumor segmentation. More specifically, it achieves the highest DSC among all the compared methods for all segmentation labels. 

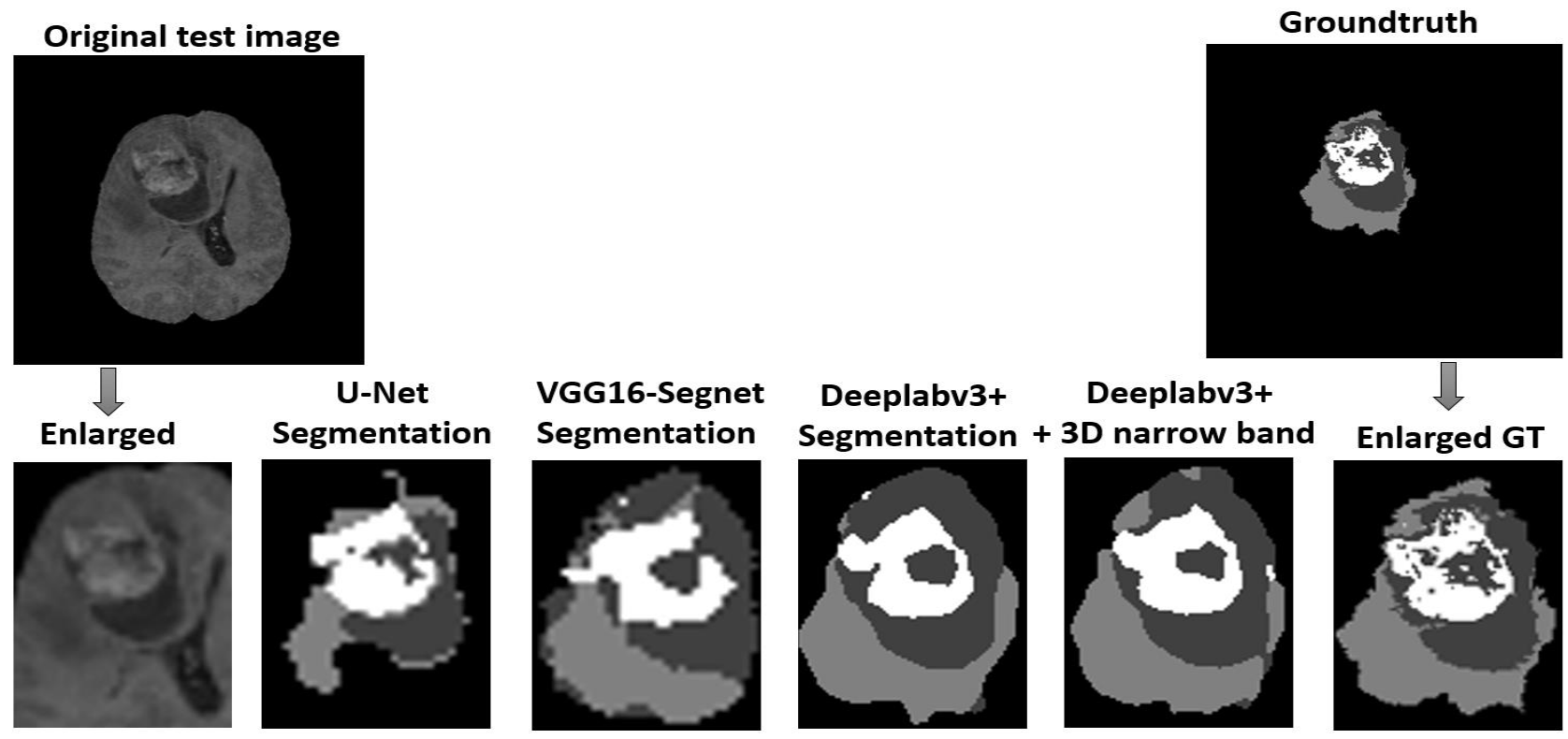

Fig.7. Results of visual segmentation for a sample test MR image, and its Ground Truth (GT) segmentation.
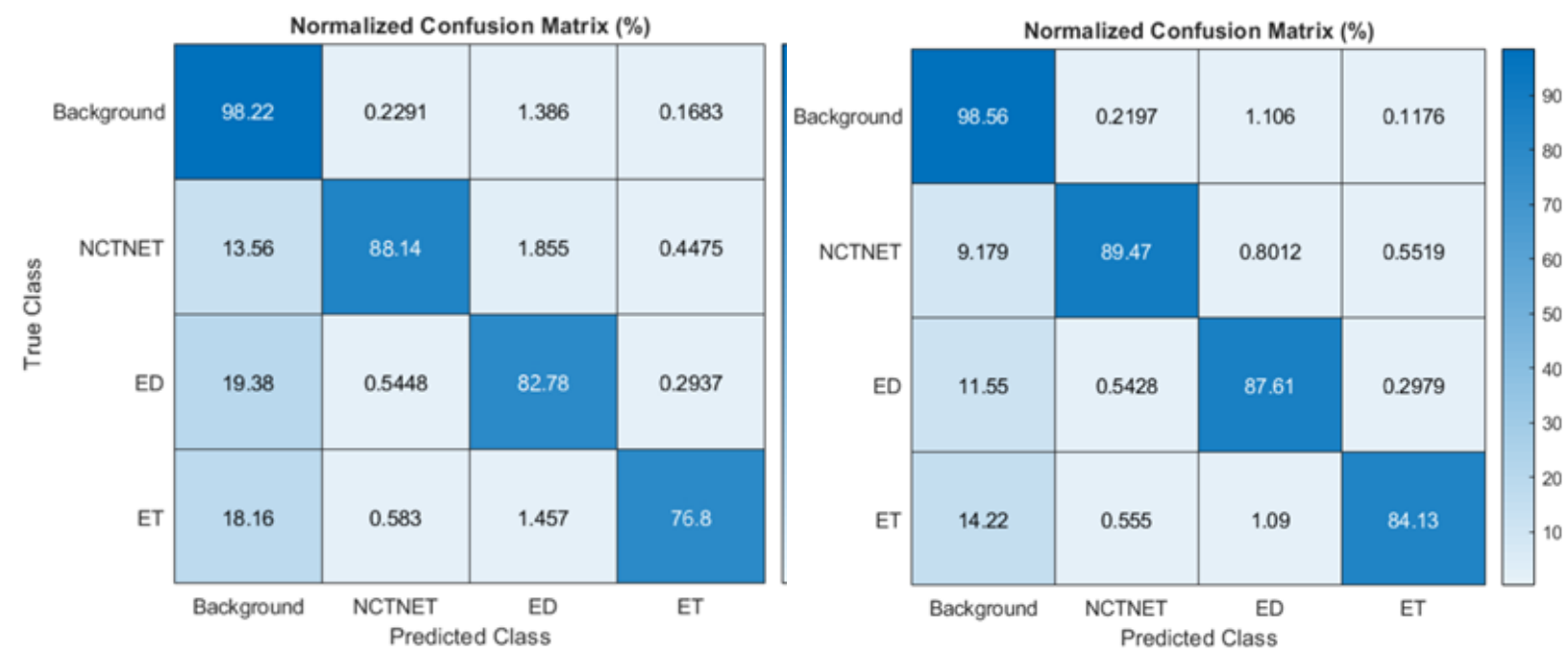

U-Net

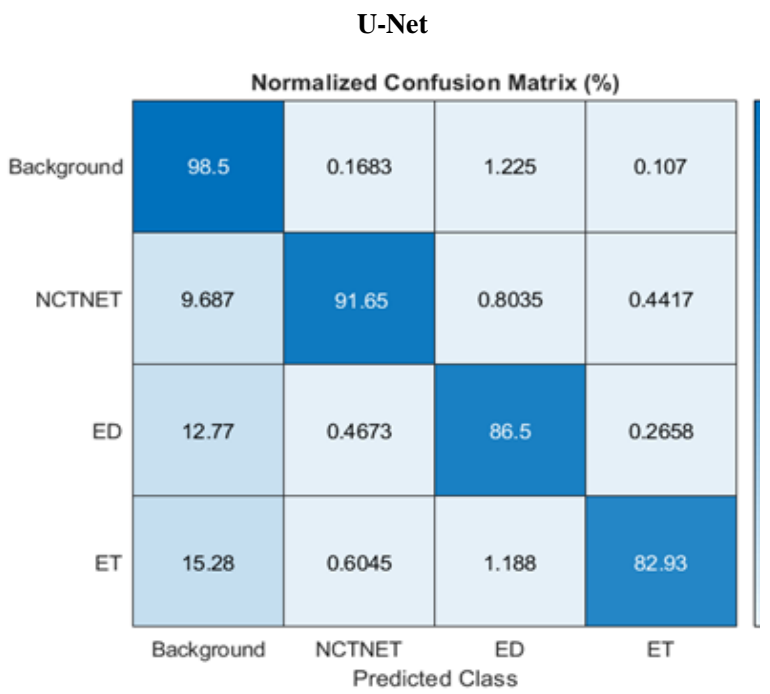

Deeplabv3+

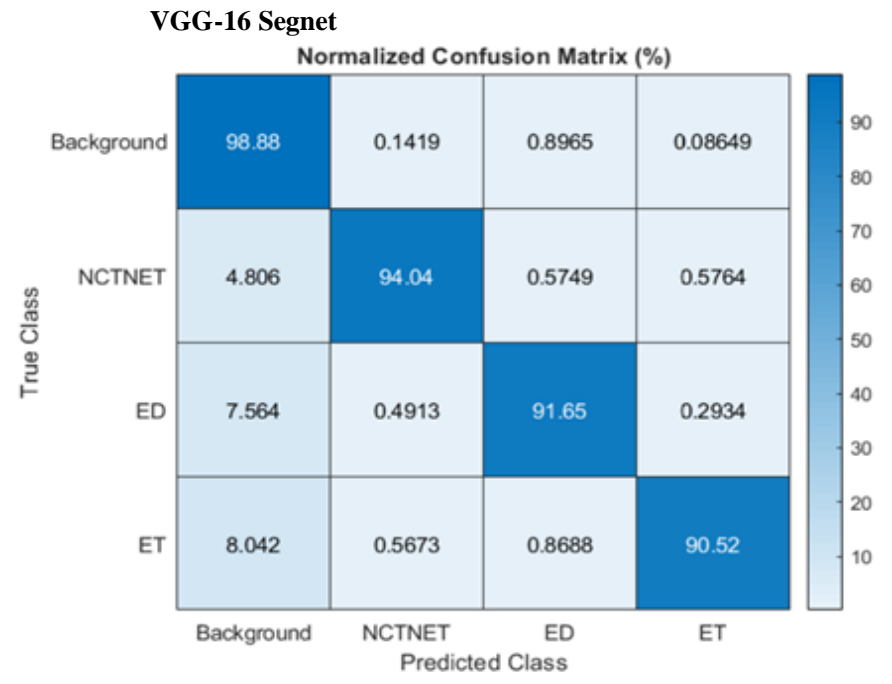

Proposed System

Fig.8. Confusion matrices of U-Net, VGG16-Segnet, Deeplabv3+ and the proposed system 
TABLE III

DICE, SENSITIVITY AND SPECIFICITY FOR EACH UTILIZED DEEP LEARNING MODELS: UNET, VGG16-SEGNET AND DEEPLABV3+

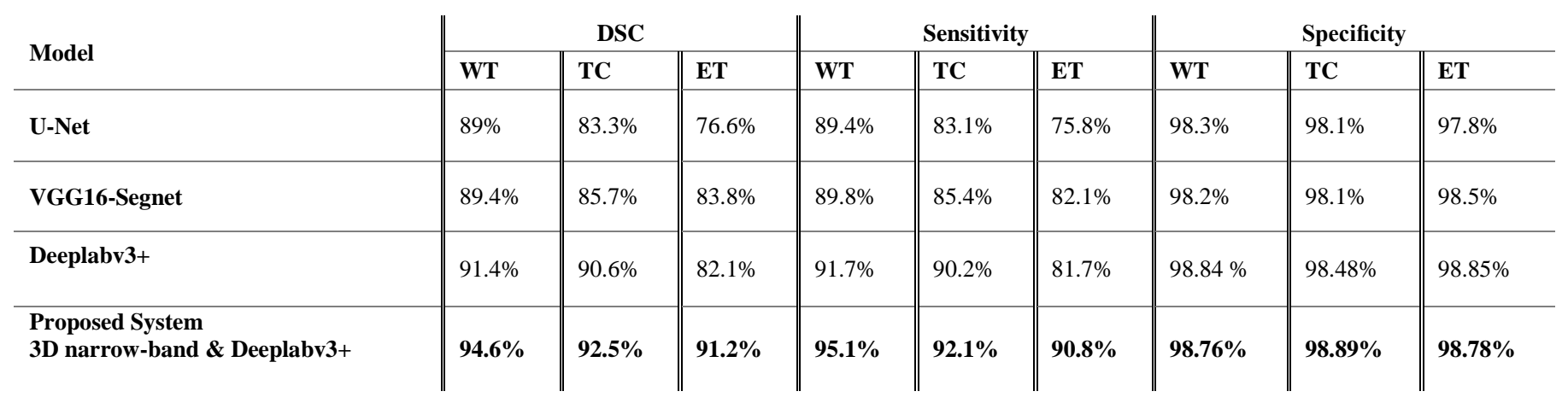

TABLE IV

COMPARISON BETWEEN THE PROPOSED SYSTEM AND THE RELATED STATE-OF-THE-ART METHODS ON THE SAME BRATS 2018 DATA.

\begin{tabular}{|c|c|c|c|c|c|}
\hline \multirow[t]{2}{*}{ Paper } & \multirow[t]{2}{*}{ Experimental setup } & \multirow[t]{2}{*}{ Method description } & \multicolumn{3}{|c|}{ DSC } \\
\hline & & & WT & TC & ET \\
\hline Roy et al. [26] & $\begin{array}{l}\text { Train size }=80 \% \\
\text { Test size }=20 \%\end{array}$ & DeepLabv3+ & $90.6 \%$ & $80.2 \%$ & $81.5 \%$ \\
\hline Rui et al. [27] & $\begin{array}{l}\text { Train size }=80 \% \\
\text { Test size }=20 \%\end{array}$ & Cascaded V-Net & $86.5 \%$ & $80.1 \%$ & $72.1 \%$ \\
\hline Ujjwal et al.[28] & $\begin{array}{c}\text { Train } \text { size }=163 \\
\text { Test size }=54\end{array}$ & modified version of 3D U-net & $93.2 \%$ & $92.0 \%$ & $80.0 \%$ \\
\hline Raghav et al. [29] & $\begin{array}{l}5 \text { cross-validation fold } \\
57 \text { patient dataset each such that each fold contains } \\
42 \text { high-grade patients and } 15 \text { low-grade patients }\end{array}$ & modified version of 3D U-net & $90.9 \%$ & $82.5 \%$ & $78.8 \%$ \\
\hline Elodie et al. [30] & $\begin{array}{l}\text { HGG and LGG } \\
\text { Train size }=90 \% \\
\text { Test size }=10 \%\end{array}$ & (VGG-16 pseudo-3D concept) & $87 \%$ & NA & NA \\
\hline Proposed system & $\begin{array}{c}\text { Train size }=\mathbf{8 0 \%} \\
\text { Test size }=20 \%\end{array}$ & 3D narrow band and Deeplabv3+ & $94.6 \%$ & $92.5 \%$ & $91.2 \%$ \\
\hline
\end{tabular}

\section{CONCLUSION}

In this paper, the proposed system for brain tumor segmentation is based on deep learning experimentations. T1c modality of MR images of BraTS 2018 database are used to evaluate the performance. Practical experiments show that DeepLabv3+ with addition of 3D narrow-band information of the tumor can achieve superior performance over the literature. In the future, other databases will be investigated to check the system robustness. Also more features will be tested to further improve the segmentation accuracy.

\section{REFERENCES}

[1] National Brain Tumor Society: Quick brain tumor facts (2020), https://braintumor.org/brain-tumor-information/brain-tumor-facts/, [Online; accessed 1-June-2020].

[2] American Cancer Society (ACS): Key statistics for brain and spinal cord tumors (2020), https://www.cancer.org/cancer/brain-spinal-cord-tumorsadults/about/key-statistics.html, [Online; accessed 1-June-2020].

[3] A. Işin, C. Direkoğlu, and M. Şah, "Review of MRI-based Brain Tumor Image Segmentation Using Deep Learning Methods," Procedia Comput. Sci., vol. 102, no. August, pp. 317-324, 2016, doi: 10.1016/j.procs.2016.09.407.

[4] E. Dandıl, M. Çakıroğlu, and Z. Ekși, "Computer-aided diagnosis of malign and benign brain tumors on MR images," in International Conference on ICT Innovations, 2014, pp. 157-166.

[5] N. J. Tustison et al., "Optimal symmetric multimodal templates and concatenated random forests for supervised brain tumor segmentation (simplified) with ANTsR," Neuroinformatics, vol. 13, no. 2, pp. 209$225,2015$.
[6] B. H. Menze et al., "The multimodal brain tumor image segmentation benchmark (BRATS)," IEEE Trans. Med. Imaging, vol. 34, no. 10, pp. 1993-2024, 2014.

[7] D. Kwon, R. T. Shinohara, H. Akbari, and C. Davatzikos, "Combining generative models for multifocal glioma segmentation and registration," in International Conference on Medical Image Computing and Computer-Assisted Intervention, 2014, pp. 763-770.

[8] Sh. Wu1, , H. Li1 and Y. Guan, "Multimodal Brain Tumor Segmentation Using U-Net," in Pre-Conference Proceedings of the 7th MICCAI BraTS Challenge, 2018, PP. 508-515.

[9] (2018) MICCAI challenge website. [Online]. Available: https://www.med.upenn.edu/sbia/brats2018/data.html

[10] M. Havaei et al., "Brain tumor segmentation with deep neural networks," Med. Image Anal., vol. 35, pp. 18-31, 2017.

[11] S. N. Shivhare, S. Sharma, and N. Singh, "An efficient brain tumor detection and segmentation in MRI using parameter-free clustering," in Machine intelligence and signal analysis, Springer, 2019, pp. 485-495.

[12] G. Gonella, E. Binaghi, P. Nocera, and C. Mordacchini, "Investigating the behaviour of machine learning techniques to segment brain metastases in radiation therapy planning," Appl. Sci., vol. 9, no. 16, p. 3335, 2019.

[13] O. Ronneberger, P. Fischer, and T. Brox, "U-net: Convolutional networks for biomedical image segmentation," in International Conference on Medical image computing and computer-assisted intervention, 2015, pp. 234-241.

[14] V. Badrinarayanan, A. Kendall, and R. Cipolla, "Segnet: A deep convolutional encoder-decoder architecture for image segmentation," IEEE Trans. Pattern Anal. Mach. Intell., vol. 39, no. 12, pp. 2481-2495, 2017

[15] L.-C. Chen, Y. Zhu, G. Papandreou, F. Schroff, and H. Adam, "Encoder-decoder with atrous separable convolution for semantic image segmentation," in Proceedings of the European conference on computer vision (ECCV), 2018, pp. 801-818. 
[16] K. Simonyan and A. Zisserman, "Very deep convolutional networks for large-scale image recognition," arXiv Prepr. arXiv1409.1556, 2014.

[17] K. He, X. Zhang, S. Ren, and J. Sun, "Deep residual learning for image recognition," in Proceedings of the IEEE conference on computer vision and pattern recognition, 2016, pp. 770-778.

[18] F. Chollet, "Xception: Deep learning with depthwise separable convolutions," in Proceedings of the IEEE conference on computer vision and pattern recognition, 2017, pp. 1251-1258.

[19] P. Tang et al., "Efficient skin lesion segmentation using separable-Unet with stochastic weight averaging," Comput. Methods Programs Biomed., vol. 178, pp. 289-301, 2019.

[20] E. Vorontsov, A. Tang, C. Pal, and S. Kadoury, "Liver lesion segmentation informed by joint liver segmentation," in 2018 IEEE 15th International Symposium on Biomedical Imaging (ISBI 2018), 2018, pp. $1332-1335$.

[21] A. Elnakib, H. E.-D. S. Moustafa, and N. Elmenabawy, “Automated Deep System for Joint Liver and Tumor Segmentation Using Majority Voting (Dept. E)," MEJ. Mansoura Eng. J., vol. 45, no. 4, pp. 30-36, 2020.

[22] G. Calderon-Auza et al., "A Teleophthalmology Support System Based on the Visibility of Retinal Elements Using the CNNs," Sensors, vol. 20, no. 10 , p. $2838,2020$.

[23] J. Zhang, Y. Xia, and Y. Zhang, "An Ensemble of Deep Neural Networks for Segmentation of Lung and Clavicle on Chest Radiographs," in Annual Conference on Medical Image Understanding and Analysis, 2019, pp. 450-458.

[24] G. Xu, H. Cao, Y. Dong, C. Yue, K. Li, and Y. Tong, "Focal Loss Function based DeepLAdam: A method for stochastic optimizationabv3+ for Pathological Lymph Node Segmentation on PET/CT," in Proceedings of the 2020 2nd International Conference on Intelligent Medicine and Image Processing, 2020, pp. 24-28.

[25] D. P. Kingma and J. Ba, "Adam: A method for stochastic optimization," arXiv Prepr. arXiv1412.6980, 2014.

[26] A. R. Choudhury, R. Vanguri, S. R. Jambawalikar, and P. Kumar, "Segmentation of brain tumors using DeepLabv3+," in International MICCAI Brainlesion Workshop, 2018, pp. 154-167.
[27] R. Hua, Q. Huo, Y. Gao, Y. Sun, and F. Shi, "Multimodal brain tumor segmentation using cascaded V-nets," in International MICCAI Brainlesion Workshop, 2018, pp. 49-60.

[28] U. Baid et al., "GBM segmentation with 3D U-Net and survival prediction with radiomics," in Pre-conference proceedings of the 7th medical image computing and computer-assisted interventions (MICCAI) BraTS Challenge, 2018, pp. 28-35.

[29] R. Mehta and T. Arbel, "3D U-Net for brain tumour segmentation," in International MICCAI Brainlesion Workshop, 2018, pp. 254-266.

[30] E. Puybareau, J. Chazalon and G. Tochon, "Glioma Segmentation In a Few Seconds Using Fully Convolutional Network and Transfer Learning," in Pre-Conference Proceedings of the 7th MICCAI BraTS Challenge, 2018, PP. $394-401$.

\section{Title in Arabic:}

تقسيم أورام المخ لصور أشعة الرنين المغناطيسى باستخدام التعليم العميق

\section{Abstract in Arabic:}

يقدم هذا البحث طريقة لتصنيف أورام المخ وتجزئتها بناءً على تحليل التعلم العميق أنقايق

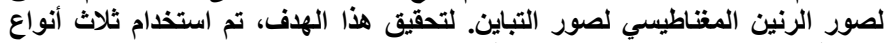

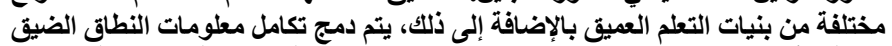

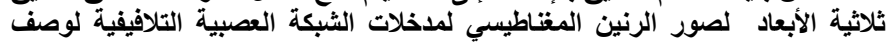

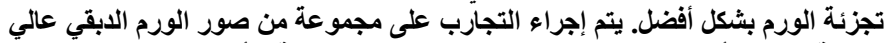

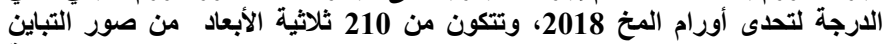

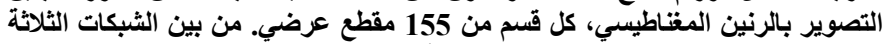

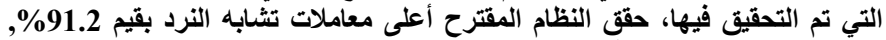

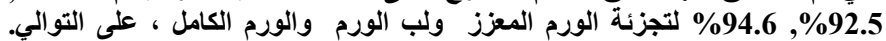
المقارنة مع الأعمال ذات الصلة تؤكد مزايا النظام المقترح. 